\title{
Pain in rheumatic diseases: how relevant is it?
}

\author{
P. Sarzi-Puttini ${ }^{1}$, F. Atzeni', F. Salaffi ${ }^{2}$ \\ ${ }^{1}$ Rheumatology Unit, L. Sacco University Hospital, Milan; \\ ${ }^{2}$ Radiology Department, Polytechnic University of the Marche, Ancona, Italy
}

\begin{abstract}
SUMMARY
Pain, a complex phenomenon influenced by a series of genetic, biological, psychological and social factors, is a major component of many rheumatological conditions and the result of physiological interactions between central and peripheral nervous system signalling $(1,2)$. It may be acute or chronic (generally defined as lasting $\geq$ three months): acute pain is often primarily attributable to inflammation and/or damage to peripheral structures (i.e. nociceptive input), whereas chronic pain is more likely to be due to input from the central nervous system (CNS) $(2,3)$.

The many different aspects of pain mean that rheumatologists and other clinicians need to have enough expertise to diagnose the type of pain correctly and treat it appropriately $(3,4)$. However, most rheumatologists receive little formal training concerning contemporary theories of pain processing or management, and this may affect the clinical results of any specific target therapy.
\end{abstract}

Key words: Pain, Rheumatic diseases, Therapy.

Reumatismo, 2014; 66 (1): 1-3

\section{TYPES OF PAIN}

$\mathrm{R}$ heumatological syndromes are generally chronic and this is often reflected in the onset and persistence of symptoms such as pain, whose physiopathological characteristics may change over time. For example, the pain associated with rheumatoid arthritis (RA) is probably nociceptive in origin when the joints are inflamed, but may gradually become centralised as it spreads throughout the body, which means it may also be simultaneously nociceptive and centralised for long periods of time (4, 5). Consequently, it may be necessary to treat (or prevent) the centralised aspects of pain while providing anti-nociceptive treatment.

\section{THE ROLE OF CENTRALISED PAIN IN CLASSIC RHEUMATIC DISEASES}

It has been known for some time that as many as $15-30 \%$ of patients with classic autoimmune or rheumatic disorders also have a co-morbid fibromyalgia (FM), once known as "secondary FM" (4). As these rates are much higher than the prevalence of FM in the general population $(2 \%)$, it seems that the pain and/or stress accompanying chronic rheumatic diseases is also capable of triggering conditions such as FM (2).

It has been shown that subsets of patients with any chronic pain condition are characterised by female gender, early life trauma, a personal or family history of chronic pain, or a personal history of other centrally mediated symptoms (insomnia, fatigue, memory problems and mood disturbances) and cognitions such as catastrophising, and that all of these factors can predict the likelihood that acute pain will become chronic $(6,7)$.

Immunological cascades may play a role in maintaining central sensitivity and chronic pain, which is increased when CNS glial cells release pro-inflammatory cytokines; the traditional dichotomy of inflammatory vs non-inflammatory pain may therefore be less appropriate than previously thought (6). $\overline{\text { Corresponding author: }}$ Piercarlo Sarzi-Puttini Rheumatology Unit L. Sacco University Hospital Via G.B. Grassi, 74 - 20127 Milano, Italy E-mail: atzenifabiola@hotmail.com piercarlo.sarziputtini@gmail.com 
MULTIFOCAL PAIN AND ANCILLARY SYMPTOMS

One of the simplest means of identifying subjects whose chronic pain has become centralised is to look for the symptoms of co-morbidities $(1,8)$. The occurrence of multifocal pain in conjunction with other centrally mediated symptoms is frequently observed in patients developing centralised pain $(8,9)$. The leading pathophysiological theory concerning concomitant clusters of somatic symptoms and higher than expected rates of mood disorders is that the abnormal centrally acting neurotransmitters that probably play a role in causing pain (e.g. low levels of norepinephrine, GABA or serotonin, and high levels of glutamate or substance P) are also involved in controlling sleep, mood and alertness (8). The best support for this hypothesis is that when centrally acting analgesics such as selective serotonin and norepinephrine re-uptake inhibitors (SSNRIs), gabapentinoids, tricyclic antidepressant or $\gamma$-hydroxybutyrate are effective in relieving chronic pain, this is frequently accompanied by improvements in other symptom domains $(1,10-12)$.

\section{IS PAIN THE SAME IN DIFFERENT RHEUMATIC CONDITIONS?}

Identifying subsets of patients affected by different rheumatic or autoimmune diseases with prominent CNS factors might also help to explain a long-standing conundrum (10). Peripherally based models clearly cannot explain much of the variance in pain, fatigue, sleep, memory problems and functional disability that is not accounted for by peripheral factors alone. For example, although the pathological focus in osteoarthritis (OA) is the joint and surrounding structures, patients with knee OA frequently experience multifocal pain in areas unaffected by OA $(4,5)$. Similarly, there are other somatic symptoms that cannot be explained on the basis of a purely peripheral condition: studies have found that fatigue is a major problem in patients with knee OA as many of them find it a more functionally limiting symptom than pain $(5,8)$. Peripherally based theories of the pathogenesis of OA, systemic lupsu erythematosus (SLE), and inflammatory arthritis simply do not explain why these other symptoms are so common and often refractory to standard therapies $(1,5)$.

\section{THE COMPLEXITY OF TREATMENT}

Pain can be most effectively treated by carefully selecting various pharmacological and non-pharmacological interventions based on the characteristics of the pain itself, disease factors, psychological coping abilities, and lifestyle factors (11). Inadequately treated chronic pain prevents many patients from taking part in everyday activities, adversely affects their working abilities, and contributes to a high rate of depression and anxiety. The chronic noncancer pain associated with rheumatic diseases is often inadequately managed (10). Paracetamol and non-steroidal antiinflammatory drugs (NSAIDs) are still used to treat of chronic rheumatic diseases, but there is increasing evidence that traditional non-selective NSAIDs and selective cyclooxygenase 2 inhibitors can have toxic effects (11-13). This is particularly important in the case of elderly patients, who are more likely to have significant problems with pain than their younger counterparts, and are at high risk of NSAID-related adverse events. If paracetamol and other nonopioid analgesics are ineffective, it may be more appropriate to administer mild opioids (codeine or tramadol) or even strong opioids, such as morphine, hydromorphone and oxycodone (13). Adequate pain relief requires an understanding of individual needs, and it has been found that non-pharmacological techniques such as cognitive behavioural therapy, biofeedback, meditation and mindfulness training, and relaxation therapy can all help patients suffering from rheumatic pain $(11,14,15)$. Furthermore, almost any form of physical activ- 
ity is essential for the global health in all patients with musculoskeletal complaints, and exercise has many beneficial effects on the physical and mental status of patients with chronic pain (14).

\section{CONCLUSIONS}

Chronic pain does not have the same relevance in all rheumatic or autoimmune diseases, and it is still unknown why pain remains localised to a specific joint or topographical area in some patients, but spreads throughout the body in others (16). Some risk factors clearly play a major role in the clinical expression of pain and related syndromes, including genetics, age, gender, co-morbidities, traumas and psychological patterns, but there no specific clinical, laboratory or neuroimaging markers that can indicate why and when a patient's localised pain will evolve into chronic widespread pain. Nevertheless, it is clear that the intensity and localisation of pain should be assessed during any clinical evaluation, and that pain of any type must always be treated in order to prevent it from becoming chronic. Finally, the treatment itself should be based on an integrated therapeutic strategy that goes beyond specific disease-related therapy, and takes into account the peripheral pattern and centralisation of pain in each individual patient, and the psychosocial factors affecting the response to pain (17). Pain may become less of a problem if we do our best to treat it promptly and appropriately.

\section{REFERENCES}

1. Stisi S, Cazzola M, Buskila D, Spath M, Giamberardino MA, Sarzi-Puttini P, et al. Italian Fibromyalgia Network. Etiopathogenetic mechanisms of fibromyalgia syndrome. Reumatismo. 2008; 60 (Suppl. 1): 25-35.

2. Sarzi-Puttini P, Atzeni F, Mease P. Chronic widespread pain or fibromyalgia? That is the question. Best Pract Res Clin Rheumatol. 2011; 25: 131-2.

3. Phillips K, Clauw DJ. Central pain mechanisms in the rheumatic diseases: future directions. Arthritis Rheum. 2013; 65: 291-302.
4. Atzeni F, Cazzola M, Benucci M, Di Franco M, Salaffi F, Sarzi-Puttini P. Chronic widespread pain in the spectrum of rheumatological diseases. Best Pract Res Clin Rheumatol. 2011; 25165-71.

5. Atzeni F, Sallì S, Benucci M, Di Franco M, Alciati A, Sarzi-Puttini P. Fibromyalgia and arthritides. Reumatismo. 2012; 64: 286-92.

6. Clauw DJ, Witter J. Pain and rheumatology: thinking outside the joint [editorial]. Arthritis Rheum. 2009; 60: 321-4.

7. Steiman AJ, Pope JE, Thiessen-Philbrook H, Li L, Barnabe C, Kalache F, et al. Non-biologic disease-modifying antirheumatic drugs (DMARDs) improve pain in inflammatory arthritis (IA): a systematic literature review of randomized controlled trials. Rheumatol Int. 2013; 33: 1105-20.

8. Goesling J, Clauw DJ, Hassett AL. Pain and depression: an integrative review of neurobiological and psychological factors. Curr Psychiatry Rep. 2013; 15: 421.

9. Alciati A, Sarzi-Puttini P, Batticciotto A, Torta R, Gesuele F, Atzeni F, et al. Overactive lifestyle in patients with fibromyalgia as a core feature of bipolar spectrum disorder. Clin Exp Rheumatol. 2012; 30: 122-8.

10. Cherubino P, Sarzi-Puttini P, Zuccaro SM, Labianca $\mathrm{R}$. The management of chronic pain in important patient subgroups. Clin Drug Investig. 2012; 32: 35-44.

11. Sarzi-Puttini P, Vellucci R, Zuccaro SM, Cherubino P, Labianca R, Fornasari D. The appropriate treatment of chronic pain. Clin Drug Investig. 2012; 32: 21-33.

12. Sarzi-Puttini P, Atzeni F, Salaffi F, Cazzola M, Benucci M, Mease PJ. Multidisciplinary approach to fibromyalgia: what is the teaching? Best Pract Res Clin Rheumatol. 2011; 25:311-9.

13. Mease PJ, Dundon K, Sarzi-Puttini P. Pharmacotherapy of fibromyalgia. Best Pract Res Clin Rheumatol. 2011; 25: 285-97.

14. Cazzola M, Atzeni F, Salaffi F, Stisi S, Cassisi G, Sarzi-Puttini P. Which kind of exercise is best in fibromyalgia therapeutic programmes? a practical review. Clin Exp Rheumatol. 2010; 28: S117-24.

15. Sarzi-Puttini P, Buskila D, Carrabba M, Doria A, Atzeni F. Treatment strategy in fibromyalgia syndrome: where are we now? Semin Arthritis Rheum. 2008; 37: 353-65.

16. Warren JW, Langenberg P, Clauw DJ. The number of existing functional somatic syndromes (FSSs) is an important risk factor for new, different FSSs. J Psychosom Res. 2013; 74: $12-7$

17. Goesling J, Clauw DJ, Hassett AL. Pain and depression: an integrative review of neurobiological and psychological factors. Curr Psychiatry Rep. 2013; 15: 421. 